\title{
Detection of hepatic maturation by Raman spectroscopy in mesenchymal stromal cells undergoing hepatic differentiation
}

\author{
Hao-Hsiang $\mathrm{Wu}^{1}$, Jennifer H. Ho $2,3,4^{*}$ and Oscar K. Lee ${ }^{1,5,6,7,8,9^{*}}$
}

\begin{abstract}
Introduction: Mesenchymal stromal cells (MSCs) are well known for their application potential in tissue engineering. We previously reported that MSCs are able to differentiate into hepatocytes in vitro. However, conventional methods for estimating the maturation of hepatic differentiation require relatively large amounts of cell samples. Raman spectroscopy (RS), a photonic tool for acquisition of cell spectra by inelastic scattering, has been recently used as a label-free single-cell detector for biological applications including phenotypic changes and differentiation of cells and diagnosis. In this study, RS is used to real-time monitor the maturation of hepatic differentiation in live MSCs.

Methods: The MSCs were cultured on the type I collagen pre-coating substrate and differentiated into hepatocytes in vitro using a two-step protocol. The Raman spectra at different time points are acquired in the range $400-3000 \mathrm{~cm}^{-1}$ and analyzed by quantification methods and principle component analysis during hepatic differentiation from the MSCs.
\end{abstract}

Results: The intensity of the broad band in the range $2800-3000 \mathrm{~cm}^{-1}$ reflects the amount of glycogen within lipochrome in differentiated hepatocytes. A high correlation coefficient between the glycogen amount and hepatic maturation was exhibited. Moreover, principle component analysis of the Raman spectra from 400 to $3000 \mathrm{~cm}^{-1}$ indicated that MSC-derived hepatocytes were close to the primary hepatocytes and were distinct from the undifferentiated MSCs.

Conclusions: In summary, RS can serve as a rapid, non-invasive, real-time and label-free biosensor and reflects changes in live cell components during hepatic differentiation. The use of RS may thus facilitate the detection of hepatic differentiation and maturation in stem cells. Such an approach may substantially improve the feasibility as well as shorten the time required compared to the conventional molecular biology methods.

Keywords: Mesenchymal stromal cells, Hepatic differentiation, Raman spectroscopy

\section{Introduction}

Inelastic scattering was first discovered by Raman [1]. When molecules are excited by incident laser light, the molecular vibration will emit scattered light. A small fraction of this scattered light has a different frequency from the incident laser light, approximately one of $10^{7}$

\footnotetext{
* Correspondence: wh9801@yahoo.com.tw; DAV47@tpech.gov.tw Hao-Hsiang $\mathrm{Wu}$ is the first author.

${ }^{2}$ Center for Stem Cell Research, Wan Fang Hospital, Taipei Medical University, Taipei 116, Taiwan

'Institute of Biophotonics, National Yang-Ming University, No. 155, Sec.2,

Linong Street, Taipei 112, Taiwan

Full list of author information is available at the end of the article
}

photons-a phenomenon called Raman scattering. The Raman shifts labeled as wave number units $\left(\mathrm{cm}^{-1}\right)$ in the Raman spectra are the difference in wavelength between the scattered and incident light in the spectrum, and exhibit the characteristics of molecular vibration [2]. For a biological sample, the complex constituents (e.g., DNA, RNA, proteins, lipids and polysaccharides) in a cell generate a 'molecular fingerprint' on the Raman spectra [3]. In recent years, high resolution Raman spectroscopy (RS) has been rapidly utilized to provide an alternative tool for the detection of different cell/tissue types [4-8], or various cellular responses including apoptosis, necrosis and cell 
differentiation $[9,10]$. Therefore, Raman spectra are able to provide unique information related to the molecular characteristics of live cells without labeling.

Stem cells are defined by their multidifferentiation potential and self-renewal capability. For tissue engineering, stem cells have provided a good means for growing new tissue and organs for transplantation [11, 12]. Mesenchymal stromal cells (MSCs) are adult stem cells which exhibit differentiation potential into bone, cartilage and adipose cells $[13,14]$. They can also be isolated from various kinds of somatic tissues and culture-expanded easily in vitro. In our previous studies, we reported that MSCs were also successfully differentiated into hepatocytelike cells in vitro [15]. Moreover, the therapeutic effect of MSC transplantation for liver failure in animal models suggests that MSCs may be useful for treating liver diseases [16-18]. However, estimating the maturation of differentiated cells before transplantation is a key challenge, and time-consuming conventional biological methods require the labeling or fixation of cells, making real-time monitoring impossible.

RS provides a faster and label-free biosensing approach to obtain cell spectra which show molecular vibrations for real-time measurement at the level of single live cells in different phenotypes. We reason that RS may be useful in detecting the stem cell commitment and differentiation. The purpose of the study is to evaluate the feasibility of using RS to measure the degree of maturation during hepatic differentiation of MSCs.

\section{Methods}

\section{Isolation of murine MSCs and hepatocytes}

Approval for these experiments was obtained from the Taipei Veterans General Hospital Institutional Animal Care and Use Committee (IACUC) regarding the use of animals prior to the commencement of the experiments. Murine MSCs were isolated from the bone marrow of 7-8-week-old Balb/c mice as previously described [19], and were cultured in low-glucose Dulbecco's modified Eagle's medium (LGDMEM; Sigma-Aldrich) supplemented with $10 \%$ fetal bovine serum (FBS; Gibco) and 1 \% PSG (Penicillin/Streptomycin/ Glutamine; Gibco). Primary murine hepatocytes were isolated from the liver of Balb/c mice euthanized by intraperitoneal injection of tribromoethanol $(240 \mathrm{mg} / \mathrm{kg})$ using a two-stage liver perfusion method reported in the literature [20, 21]. After perfusion, the liver was cut, the gall bladder was removed, and the liver was transferred to a new dish. The liver capsule was disrupted using tweezers, and the cells were released in a culture medium consisting of DMEM/ F12 (Gibco) supplemented with $10 \%$ FBS (Gibco) and $1 \%$ PSG. The cell suspension was filtered using $70-\mu \mathrm{m}$ strainers. The hepatocytes and nonparenchymal cells were separated by centrifugation at $50 \mathrm{~g}$ for 5 minutes. The hepatocytes pellet was then washed twice in the culture medium and the hepatocytes were cultured on type I collagen pre-coated cover glass.

\section{Hepatic differentiation of MSCs}

MSCs were cultured on type I collagen (BD falcon) precoated cover glass, and then treated with step 1 of the hepatic differentiation medium consisting of Iscove's modified Dulbecco's medium (IMDM; Sigma-Aldrich) supplemented with $20 \mathrm{ng} / \mathrm{mL}$ hepatocyte growth factor, $10 \mathrm{ng} / \mathrm{mL}$ fibroblast growth factor-2, $0.61 \mathrm{~g} / \mathrm{L}$ nicotinamide and $1 \%$ PSG for the first week. Then the culture condition was changed for step 2 treatment with the hepatic differentiation medium consisting of IMDM supplemented with $20 \mathrm{ng} / \mathrm{mL}$ oncostatin $\mathrm{M}, 50 \mathrm{mg} / \mathrm{mL} \mathrm{ITS}^{+}$, $1 \mu \mathrm{mol} / \mathrm{L}$ dexamethasone and $1 \%$ PSG.

\section{Quantitative polymerase chain reaction}

The total RNA of the MSCs was collected and quantified at different time points of hepatic differentiation. The mRNA was reverse-transcribed to CDNA by MMLV reverse transcriptase (EPICENTRE ${ }^{\circ}$ Biotechnologies). The 10 ng cDNA in the total volume was analyzed by quantitative polymerase chain reaction (PCR).

\section{Albumin immunostaining}

The cells were fixed using $3.7 \%$ formaldehyde for 10 minutes, and permeablized with $0.1 \%$ triton X-100 for 10 minutes. The cells were incubated with the primary antibody (R\&D MAB1455) overnight at $4{ }^{\circ} \mathrm{C}$ and then incubated with secondary antibody (abcam ab150113) for 1 hour and washed with phosphate-buffered saline (PBS).

\section{Periodic acid-Schiff staining}

The cells were fixed using $3.7 \%$ formaldehyde for $10 \mathrm{mi}-$ nutes, and washed with PBS three times. The cells were then permeablized using $0.1 \%$ triton X-100 for 10 minutes. Subsequently, the cells were treated with $1 \%$ periodic acid for 5 minutes, and washed with $\mathrm{H}_{2} \mathrm{O}$. Finally, they were treated with Schiff's fuchsin-sulfite solution for 1 hour, washed lightly with $\mathrm{H}_{2} \mathrm{O}$ and dried.

\section{Glycogen storage assay}

For quantitation of glycogen storage, the cells were lysed with $\mathrm{H}_{2} \mathrm{O}$, and boiled for 10 minutes. The extract was used to quantify the glycogen using the Glycogen Colorimetric/ Fluorometric Assay Kit (BioVision).

\section{Urea production assay}

The urea production of MSCs after 3 weeks of hepatic differentiation was investigated. Cytoplasmic protein was extracted to analyze the urea production using a Urea Colorimetric Assay Kit (BioVision). 


\section{Diastase treatment}

The cells were fixed using $3.7 \%$ formaldehyde for 10 minutes, and washed with PBS three times. The cells were then permeablized using $0.1 \%$ triton $\mathrm{X}-100$ for 10 minutes and washed by PBS. Subsequently, they were treated with $1 \mathrm{mg} / \mathrm{ml}$ diastase (Sigma) at $37^{\circ} \mathrm{C}$ for 1 hour and washed by PBS.

\section{Raman spectroscopy and data acquisition}

A Labram HR 800 Raman spectroscope (HORIBA Scientific, Japan) used He-Ne laser to excite the samples at $632.8 \mathrm{~nm}$ with $17 \mathrm{~mW}$ power. The spectroscope was equipped with an Olympus BX40, a 60x water immersion M-Plan objective $(\mathrm{NA}=0.9)$, and a liquid nitrogen-cooled CCD two-dimensional array detector. The cells were seeded on cover glasses precoated with type I collagen, and the cover glasses were placed on top of mounting glasses with cells facing upwards. Then IMDM media were dropped on the cover glasses for the purpose of detection by a 60x water immersion M-Plan objective of Raman spectroscopy. Then the position for laser excitation in the monitor was chosen randomly for detecting, and the Raman spectra were acquired in the Raman shift range $400-3000 \mathrm{~cm}^{-1}$ with an integration time of 30 seconds. Cell number for data acquisition is over 10 in every measurement. The raw spectra data were nonlinearly smoothed, baseline corrected, and analyzed using LabSpec software.

\section{Principal component analysis}

The Raman spectra $\left(400-3000 \mathrm{~cm}^{-1}\right)$ of the primary hepatocytes, undifferentiated MSCs, and MSC-derived hepatocytes were further processed by principal component analysis (PCA) using the Raman Processing (RB) program written in MATLAB developed by the SSIM/CARES research group at Wayne State University [22].

\section{Statistical analysis}

The quantitative data were expressed as mean \pm standard deviation. Statistical significance was determined by $t$ test ( $p=0.05)$ or one-way analysis of variance with Tukey's post-hoc test $(p=0.05)$ and different alphabetical letters indicate different levels of statistical significance at $95 \%$ confidence intervals.

\section{Results and discussion}

\section{In vitro hepatic differentiation of MSCs}

To test the efficiency of hepatic differentiation of MSCs, cells were cultured on type I collagen coated cover glass and were induced into hepatocytes using a previously reported protocol [15]. The cells changed in shape from fibroblast-like to cuboidal, and then became hepatocyte- like in appearance (Fig. 1a, b, and c). Albumin, the most abundant blood plasma protein secreted by hepatocytes, was measured in the MSC-derived hepatocytes using immune-staining. The results indicated that albumin expression apparently increased 3 weeks after hepatic induction (Fig. 1d and e). The mRNA expression levels of liver-specific genes investigated by quantitative PCR revealed that albumin, $\alpha$-fetoprotein, cytokeratin 18 , hepatic nuclear factor 4 , tyrosine aminotransferase, glucose 6 phosphatase, and metabolism-related cytochrome P450 enzymes (Cyp1a2, Cyp2b10, and Cyp2f2) were highly upregulated in the MSCs after hepatic induction, while cytokeratin 19 as a cholangiocyte marker was downregulated (Fig. 1f). In addition, the functional assays including glycogen storage and urea production were further investigated in the MSC-derived hepatocytes. Periodic acid-Schiff (PAS) staining and quantitative assay showed that, compared to undifferentiated MSCs, glycogen storage in the MSC-derived hepatocytes was significantly increased (Fig. $1 \mathrm{~g}$ and h). Urea production was also elevated in the MSC-derived hepatocytes (Fig. 1i). These results indicated that the MSCs were efficiently differentiated into hepatocytes.

\section{Raman spectra of lipochrome in the MSC-derived hepatocytes}

During hepatic differentiation, an increase in PAS staining-positive granules in the cytoplasm of MSCderived cells was noted (Fig. 2). PAS staining is used to detect polysaccharides such as glycogen; also, glycoproteins, glycolipids, lipids, and phospholipids are positive under PAS staining. In hepatocytes, the granules are termed lipochrome (lipofuscin) which is PAS staining-positive and composed of lipid-containing residues of lysosomal digestion. Although lipids and proteins are the main constituents in lipochrome, a small amount of carbohydrate (4-7 \%) is also found in lipochrome content [23], and glycogen is the major form of carbohydrate stored in hepatocytes. In rat liver, more than $10 \%$ of cellular glycogen is located within lysosome [24]. Normally, the mean size of lipochrome is $1 \mu \mathrm{m}$, but larger granules are also encountered [25]. Moreover, the increased formation and size of lipochrome is reported to be associated with aging [26-29]. However, lipochrome is frequently found in postmitotic cells [30] and is also found in newborn human liver [25]. The maturation of hepatocytes was reported by in vitro hepatic induction of hepatic progenitor cells from fetal mouse liver, which showed increased PAS stainingpositive granules along with induction time [31]. The above evidence indicates that it is possible to use lipochrome formation as an index of maturation level during hepatic differentiation of MSCs. To investigate Raman spectra between the MSC-derived hepatocytes and 


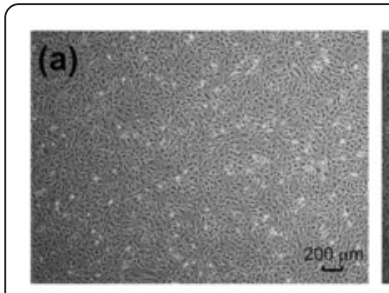

(f)
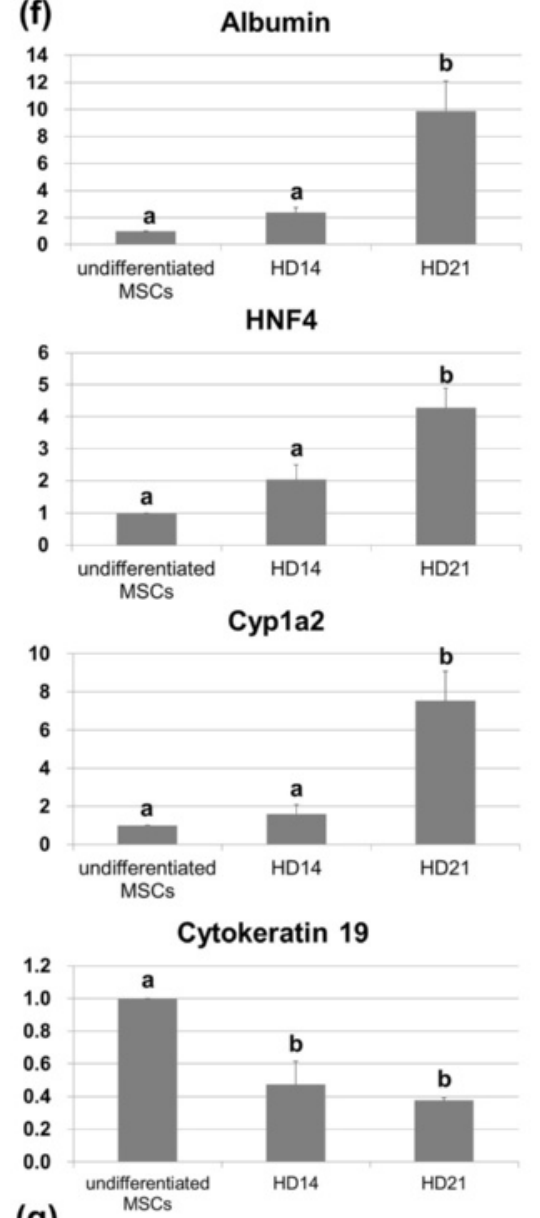

(g)

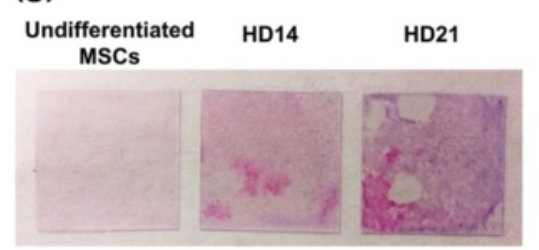

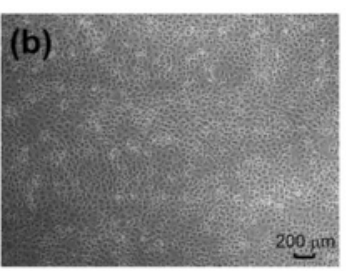

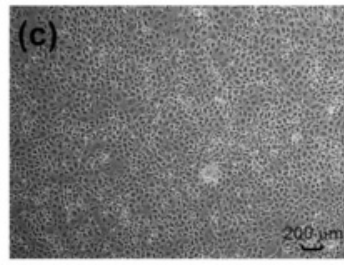

$\alpha$ - fetoprotein
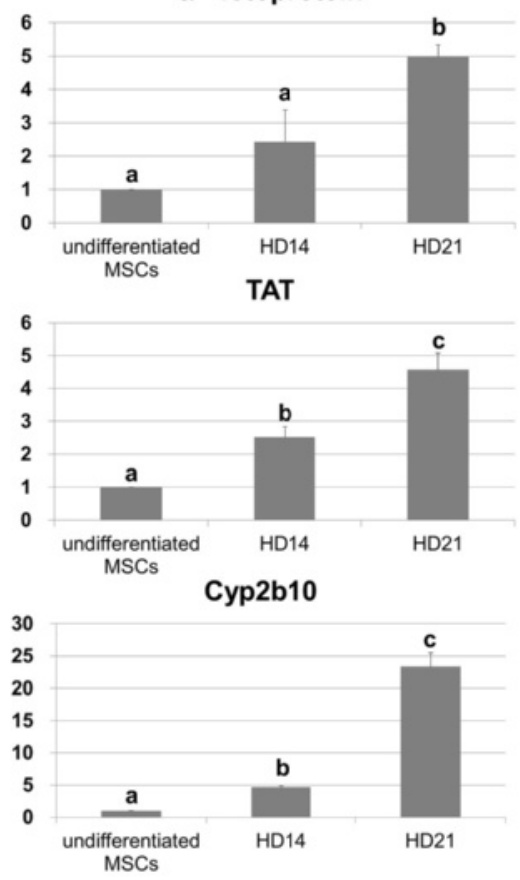

MSCs
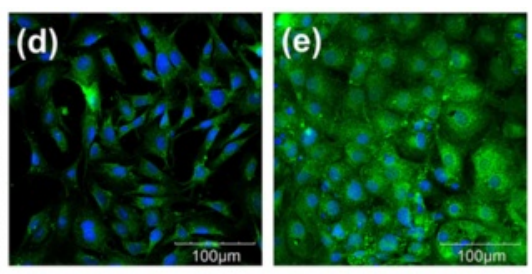

Cytokeratin 18
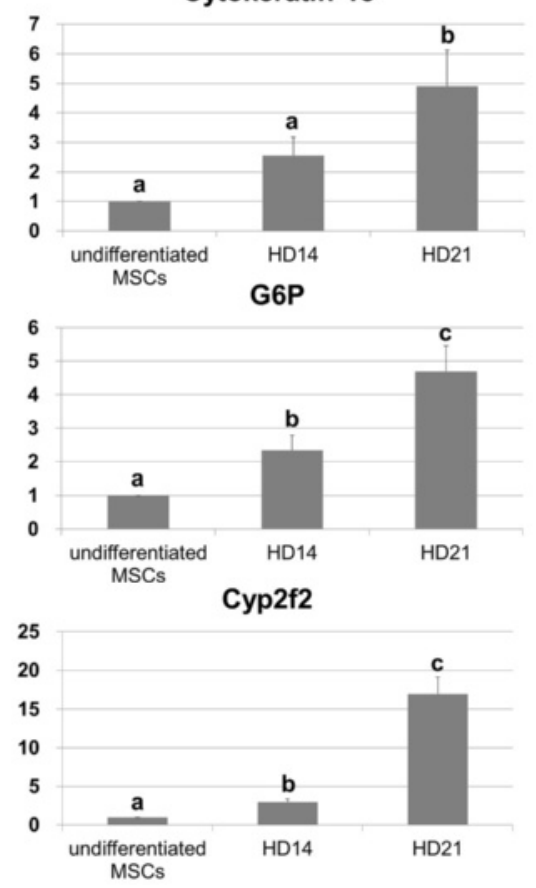

(i)

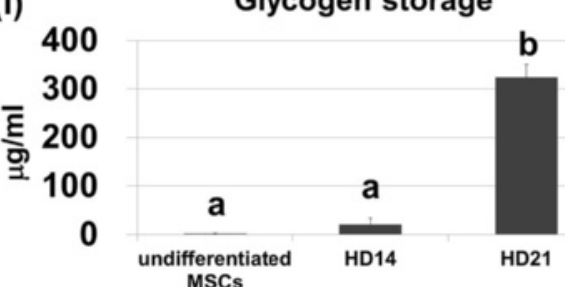

Fig. 1 Hepatic differentiation of MSCs on cover glasses precoated with type I collagen. The MSCs were cultured on cover glasses pre-coated with type I collagen, and differentiated into hepatocytes. Cell morphology of $\mathbf{a}$ undifferentiated MSCs, $\mathbf{b}$ hepatic differentiation at day 14 and $\mathbf{c}$ hepatic differentiation at day 21. Albumin staining of $\mathbf{d}$ undifferentiated MSCs and e differentiated cells. $\mathbf{f}$ The mRNA expression of MSC-derived hepatocytes. The mRNA expression of liver-specific genes was investigated on days 14 (HD14) and 21 (HD21) of hepatic differentiation as well as in undifferentiated MSCs. $\mathbf{g}$ PAS staining of the undifferentiated MSCs, and at days 14 and 21 of hepatic differentiation. Quantification of $\mathbf{h}$ glycogen storage and $\mathbf{i}$ urea production during hepatic differentiation from the MSCs. Groups with different alphabetical letters are significantly different, whereas groups with the same alphabetical letters are not; ${ }^{*} p<0.05,{ }^{* *} p<0.01$. G6P Glucose 6 phosphatase, HNF4 Hepatic nuclear factor 4 , MSC Mesenchymal stromal cell, TAT Tyrosine aminotransferase 

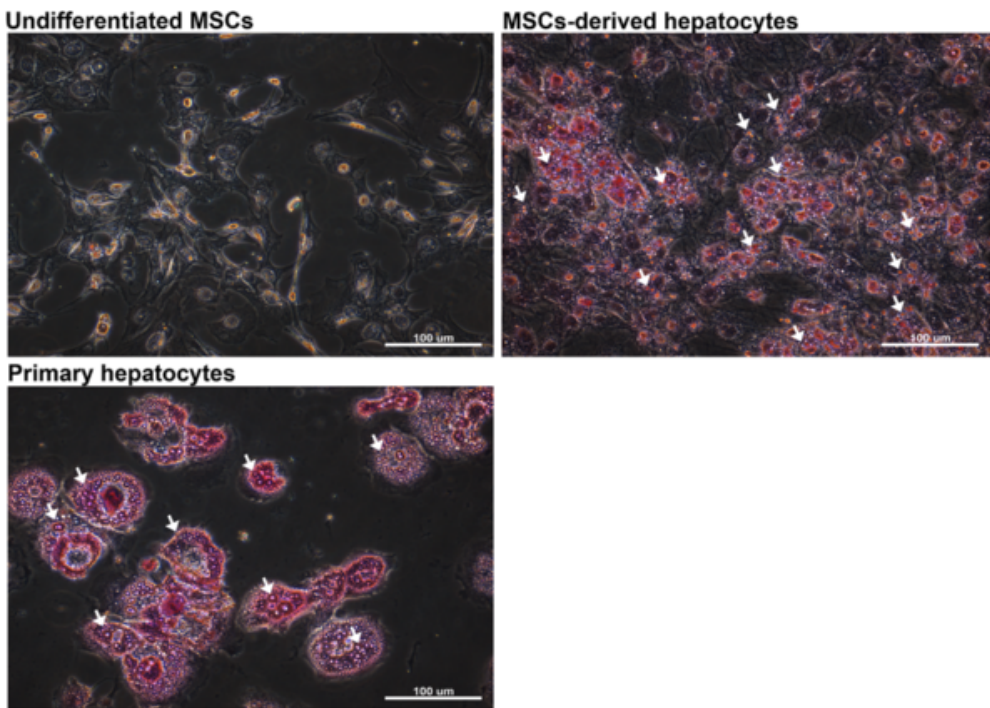

Fig. 2 The lipochrome formation in cytoplasm increased in the MSC-derived hepatocytes and primary hepatocytes. The undifferentiated MSCS, MSC-derived hepatocytes and primary hepatocytes were stained with PAS staining. The white arrows indicate the PAS staining-positive granules

primary hepatocytes, the spectra were recorded in the range $400-3000 \mathrm{~cm}^{-1}$ and the assignment of the Raman shifts highlighted in Fig. 3 are summarized in Table 1 [3235]. The spectra of lipochrome and nonlipochrome cytoplasmic position were investigated in the MSC-derived hepatocytes and primary hepatocytes. The lipochrome was not observed in the undifferentiated MSCs (Fig. 3a). Lipochrome spectra in the MSC-derived hepatocytes and primary hepatocytes both exhibited a broad band in the range $2800-3000 \mathrm{~cm}^{-1}$ with two main peaks (2852/ $2898 \mathrm{~cm}^{-1}$ ) indicating a $\mathrm{CH}_{2}$ symmetric stretch/CH stretch (Fig. $3 \mathrm{~b}$ and c). In addition to the broad band, the MSC-derived hepatocytes and primary hepatocytes also have peaks of $1301-1305 \mathrm{~cm}^{-1}$ (C-H vibration, $\mathrm{CH}_{2}$ deformation, $\mathrm{CH}_{3}$ and $\mathrm{CH}_{2}$ twisting), $1436-1439 \mathrm{~cm}^{-1}\left(\mathrm{CH}_{2}\right.$ scissoring and $\mathrm{CH}_{2}$ deformation), and $1655-1680 \mathrm{~cm}^{-1}$ (Amide I) in the lipochrome spectra. However, the peaks at $1076 \mathrm{~cm}^{-1}$ for $\mathrm{v}(\mathrm{C}-\mathrm{C})$ or $\mathrm{v}(\mathrm{C}-\mathrm{O}), 1263 \mathrm{~cm}^{-1}$ for triglycerides, and $1750 \mathrm{~cm}^{-1}$ for $\mathrm{v}(\mathrm{C}=\mathrm{C})$ were only found in the lipochrome of the primary hepatocytes (Fig. 3c). In the nonlipochrome cytoplasmic position, both the MSCderived hepatocytes and primary hepatocytes expressed a broad band in the range $2800-3000 \mathrm{~cm}^{-1}$ indicated as $v(\mathrm{C}-\mathrm{H})$ as compared with the undifferentiated MSCs in Fig. 3. In the primary hepatocytes, the spectra intensity of the different sized lipochromes was found to increase with lipochrome size (Fig. 3d). Also, lipochromes of large size were observed in the primary hepatocytes, which are harvested from adult mice. Therefore, the lipochrome formation was found during hepatic differentiation from MSCs, and the vibration of the chemical bonding shown in the Raman spectrum of lipochrome could use for estimating maturation during hepatic differentiation.

\section{Real-time monitoring of the maturation of MSC-derived hepatocytes by RS}

Time-dependent Raman spectra were measured to monitor the maturation of hepatic differentiation from the MSCs (Fig. 4a). The Raman spectra of primary hepatocytes were used as a reference and exhibited peaks at $968 \mathrm{~cm}^{-1}$, $1078 \mathrm{~cm}^{-1}, 1264 \mathrm{~cm}^{-1}, 1303 \mathrm{~cm}^{-1}, 1439 \mathrm{~cm}^{-1}, 1660 \mathrm{~cm}^{-1}$, and $1750 \mathrm{~cm}^{-1}$, and a broad band at $2800-3000 \mathrm{~cm}^{-1}$ composed of peaks at $2853 \mathrm{~cm}^{-1}$ and $2899 \mathrm{~cm}^{-1}$. Figure $4 \mathrm{~b}$ magnifies the ranges at $400-565 \mathrm{~cm}^{-1}, 1400-1500 \mathrm{~cm}^{-1}$, and $2700-3000 \mathrm{~cm}^{-1}$. The broad band intensity in the range $400-565 \mathrm{~cm}^{-1}$ was constant in the IMDM medium, undifferentiated MSCs, primary hepatocytes, and during the hepatocyte differentiation, indicating that signals in the range 400-565 $\mathrm{cm}^{-1}$ resulted from the background signal of the type I collagen coating the substrate. During the hepatic differentiation of the MSCs, the broad band intensity (2800-3000 $\left.\mathrm{cm}^{-1}\right)$ increased at day 10 and day 14 compared with the undifferentiated MSCs. In addition, a peak of $1439 \mathrm{~cm}^{-1}$ was detected at day 14 of the hepatic differentiation from the MSCs. However, the broad band intensity (2800-3000 $\left.\mathrm{cm}^{-1}\right)$ and peak $\left(1439 \mathrm{~cm}^{-1}\right)$ in the MSC-derived hepatocytes were lower than that for the primary hepatocytes. Because of the positive correlation between the broad band intensity $\left(2800-3000 \mathrm{~cm}^{-1}\right)$ and the duration of induction, we speculate that the signals at $2800-3000 \mathrm{~cm}^{-1}$ could be used to monitor the maturity of hepatic differentiation from the MSCs.

As shown in Fig. 2, lipochromes were positive for PAS staining in the MSC-derived hepatocytes. To further investigate which signal of the Raman spectra was from glycogen, MSC-derived hepatocytes were treated with diastase to catalyze the glycogen, and then the change in 
(a)

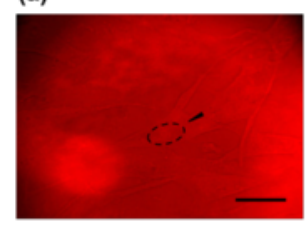

(b)

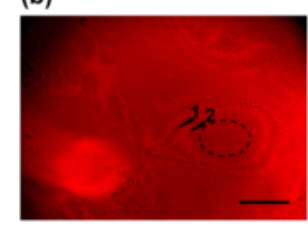

(c)

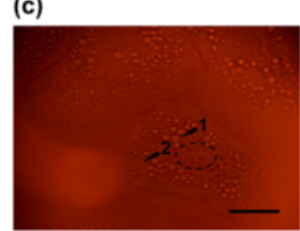

(d)

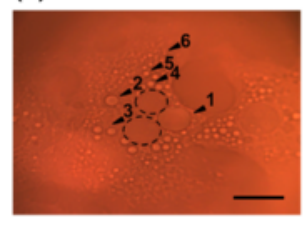

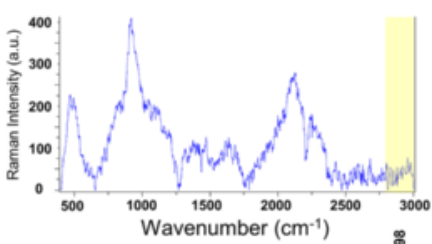

1

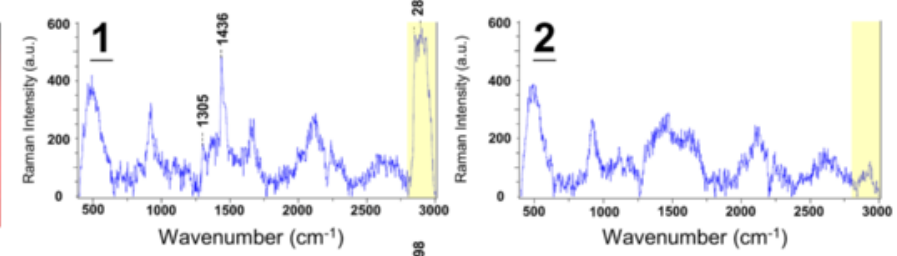

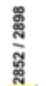
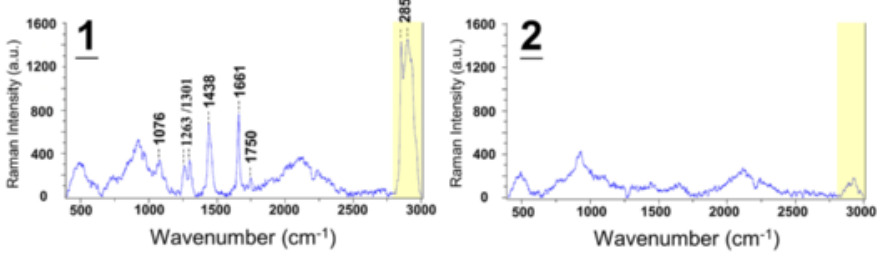

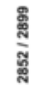

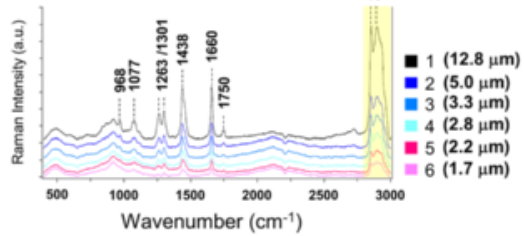

Fig. 3 Raman spectra of lipochromes in the cytoplasm of the MSC-derived hepatocytes. The Raman spectra of $\mathbf{a}$ undifferentiated MSCs, $\mathbf{b}$ MSC-derived hepatocytes (1, lipochrome; 2, nonlipochrome cytoplasm), and c primary hepatocytes (1, lipochrome; 2, nonlipochrome cytoplasm) were investigated in $400-3000 \mathrm{~cm}^{-1}$. d Raman spectra of the lipochromes with different sizes (1-6) in the cytoplasm of the primary hepatocytes. The number in parentheses indicates the diameter of lipochromes. The black arrows indicate the position of laser excitation and the circle with the dotted line indicates the nucleus. Scale bars $=20 \mu \mathrm{m}$

Raman signal was investigated. As shown in Fig. 5a, the broad band at $2800-3000 \mathrm{~cm}^{-1}$ in MSC-derived hepatocytes could no longer be detected after diastase treatment. Subsequently, to investigate whether amorphous glycogen also causes $2800-3000 \mathrm{~cm}^{-1}$ Raman shift, $10 \%$ glycogen solution was tested. The result showed that the glycogen solution also caused Raman shift $\left(2800-3000 \mathrm{~cm}^{-1}\right)$ compared to $\mathrm{H}_{2} \mathrm{O}$ control (Fig. 5b). These results indicate that the signal was indeed derived from glycogen formation during hepatocyte differentiation.

Table 1 Assignment of the Raman shifts

\begin{tabular}{ll}
\hline Raman shift & Assignments \\
\hline $968 \mathrm{~cm}^{-1}$ & Lipids \\
$1076-1078 \mathrm{~cm}^{-1}$ & $\mathrm{U}(\mathrm{C}-\mathrm{C})$ or $\mathrm{U}(\mathrm{C}-\mathrm{O})$ \\
$1263-1264 / 1301-1303 \mathrm{~cm}^{-1}$ & Triglycerides (fatty acids), $=\mathrm{C}-\mathrm{H} / \mathrm{C}-\mathrm{H}$ vibration, $\mathrm{CH}_{2}$ deformation, $\mathrm{CH}_{3}$ and $\mathrm{CH}_{2}$ twisting \\
$1436-1439 \mathrm{~cm}^{-1}$ & $\mathrm{CH}_{2}$ scissoring, $\mathrm{CH}_{2}$ deformation \\
$1655-1680 \mathrm{~cm}^{-1}$ & Amide I \\
$1750 \mathrm{~cm}^{-1}$ & $\mathrm{U}(\mathrm{C}=\mathrm{C})$ \\
$2852-2881 / 2899-2907 \mathrm{~cm}^{-1}$ & $\mathrm{CH}$ symmetric stretch/CH stretch (lipids) \\
$2800-3000 \mathrm{~cm}^{-1}$ & $\mathrm{U}(\mathrm{C}-\mathrm{H})$ \\
\hline
\end{tabular}




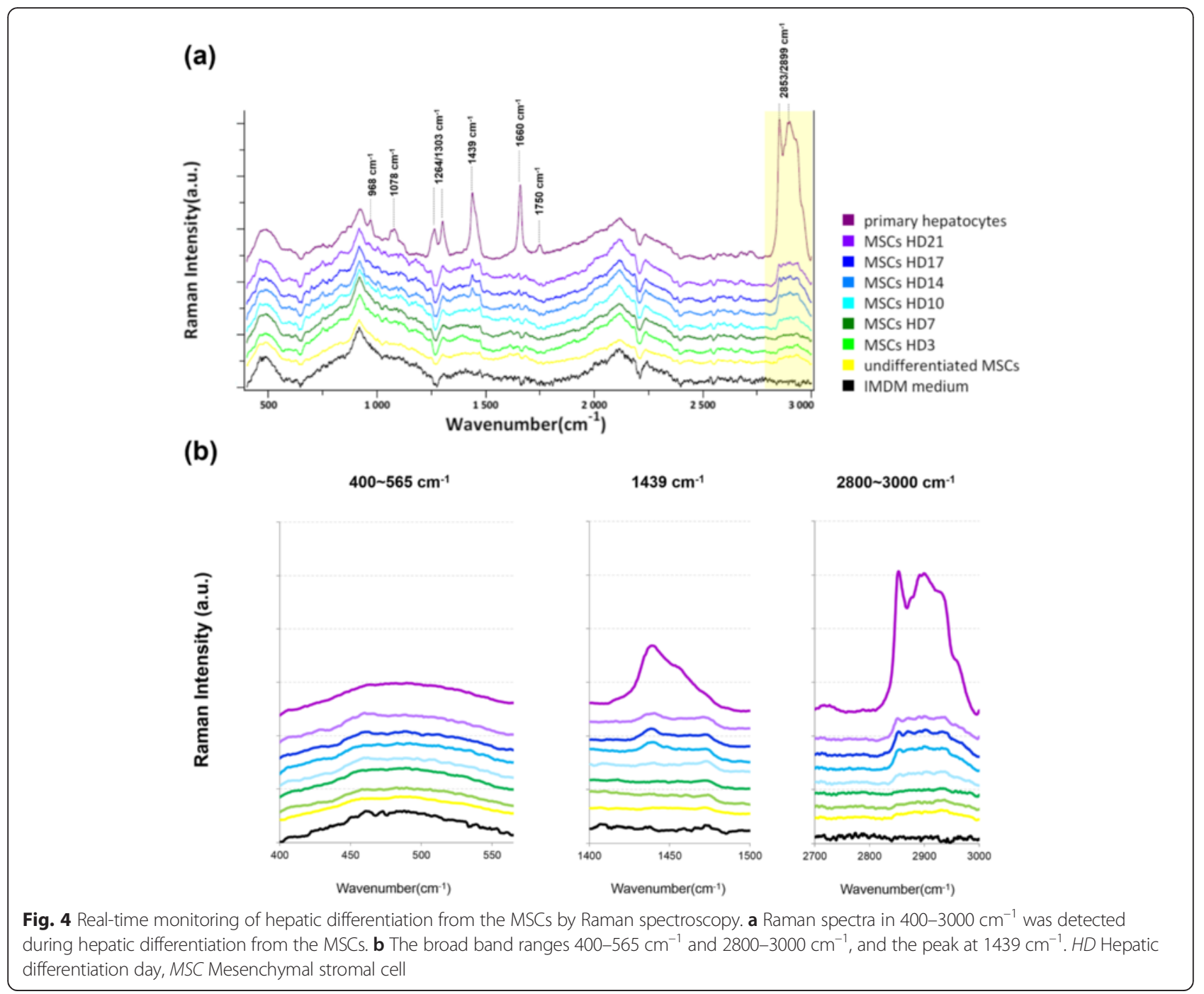

\section{Quantification of maturation level during hepatic differentiation from the MSCs}

To quantify the Raman spectra results, the integral area of the broad band $\left(2800-3000 \mathrm{~cm}^{-1}\right)$ was calculated and normalized according to the background signal of the broad band (400-565 $\mathrm{cm}^{-1}$ ) (Fig. 6a). At day 3 of hepatic differentiation, the ratio showed no significant difference as compared to the undifferentiated MSCs, but the ratio increased significantly at days $10,14,17$, and 21. Furthermore, the ratio from day 7 to day 17 increased linearly and the correlation coefficient also exhibited a high degree of correlation with the induction time $\left(R^{2}=0.996\right)$ (Fig. 6b). The correlation coefficient from day 7 to day 17 indicated a high correlation between qualification of signals at $2800-3000 \mathrm{~cm}^{-1}$ and the maturation level during hepatic differentiation from the MSCs. Furthermore, it indicates gradual maturation in hepatic phenotype. The results indicate high correlation between the qualification of signals at $2800-3000 \mathrm{~cm}^{-1}$ and the maturation level during hepatic differentiation from the MSCs.

\section{PCA of Raman spectra in the MSC-derived hepatocytes}

To investigate the differences and similarities among the undifferentiated MSCs, MSC-derived hepatocytes, and primary hepatocytes, the Raman spectra were analyzed by PCA. As shown as Fig. 7, component scores of the undifferentiated MSCs and MSCs under hepatic differentiation for 7 and 21 days separated into three subgroups, indicating the different stages of hepatic differentiation. It was found that the MSC-derived hepatocytes at day 7 and day 21 were closer to the primary hepatocytes than the undifferentiated MSCs. The results indicate that the spectra of undifferentiated MSCs changed their Raman spectra signature to ones similar to the primary hepatocytes during hepatic differentiation. 
(a)
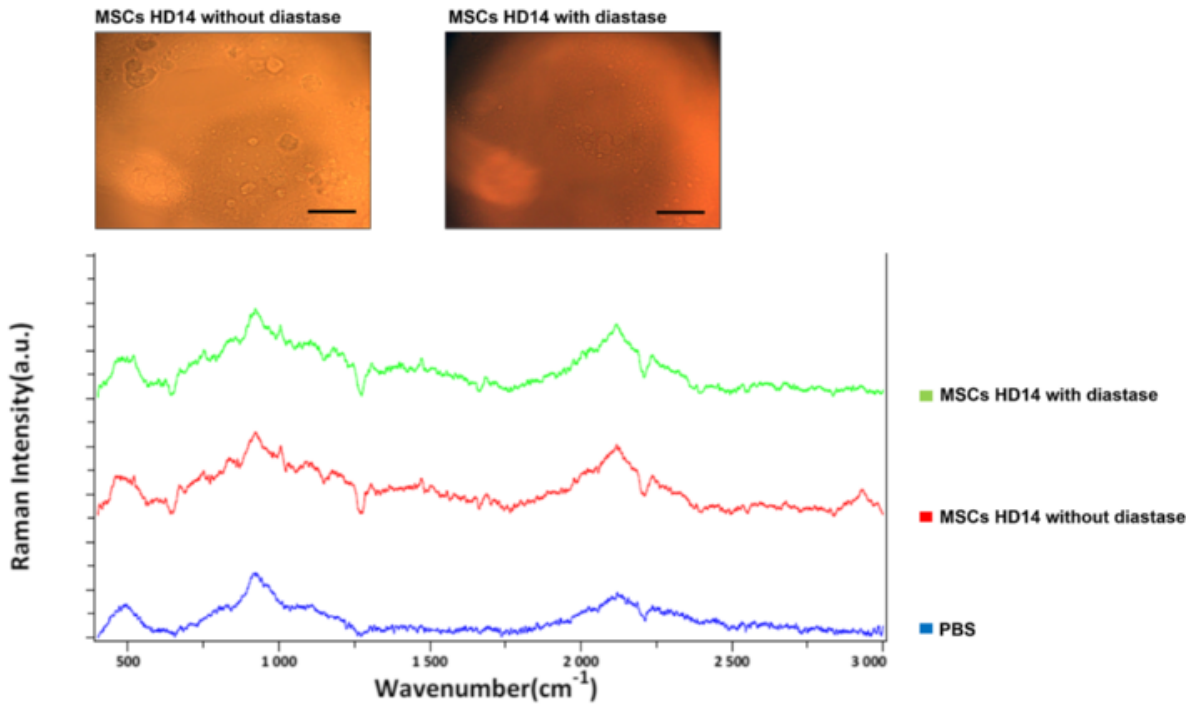

(b)

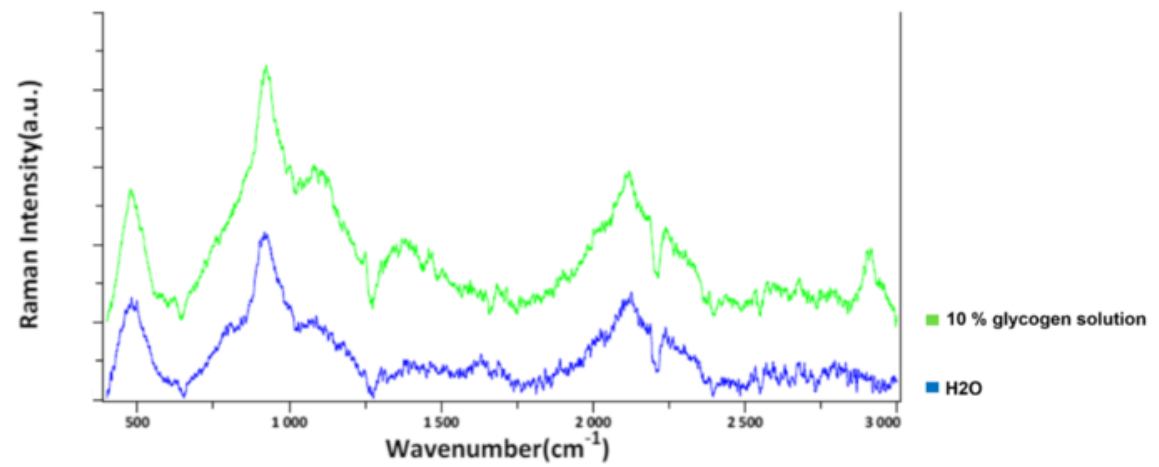

Fig. 5 Raman spectra of the MSC-derived hepatocytes after glycogen digestion. a The cell morphology (top) and Raman spectra (bottom) of the MSC-derived hepatocytes at day 14 of hepatic differentiation with or without diastase treatment for glycogen catalysis. b The Raman spectra of $10 \%$ glycogen solution. Scale bars $=20 \mu \mathrm{m}$. HD Hepatic differentiation day, MSC Mesenchymal stromal cell, PBS Phosphate-buffered saline

A conventional molecular biology approach, such as quantitative PCR or immunostaining, to measure the maturation level of hepatic differentiation requires sacrificing a large number of cells, which makes real-time monitoring on live cells during hepatic differentiation impossible. In this study, we demonstrate that RS is a powerful tool for monitoring real-time molecular information at a single-cell level during hepatic differentiation from MSCs. The spectra of Raman scattering in MSC-derived hepatocytes during differentiation provide a unique fingerprint at each stage, which reflects the characteristics of molecular vibration composed of extremely complex cell components. RS can be therefore used for biosensing and to quantitatively evaluate the maturation level of hepatic differentiation from the MSCs by the signals at $2800-3000 \mathrm{~cm}^{-1}$.
The structure of multibranched polysaccharide in the glycogen structure contains a large amount of glycosidic bonds. Furthermore, Raman shift (2800-3000 cm $\left.{ }^{-1}\right)$ is also reported to represent the $\mathrm{CH}$ stretch from glyosidic linkage [36]. We show that the broad band (2800-3000 $\mathrm{cm}^{-1}$ ) assigned for $v(\mathrm{C}-\mathrm{H})$ increased in intensity with hepatic differentiation (Fig. 4), indicating that the broad band signal is derived from glycosidic bonds of the glycogen structure in the MSCderived hepatocytes by digesting glycogen (Fig. 5). Moreover, the real-time evaluation of the maturity level during hepatic differentiation of MSCs could be achieved by quantitative analysis of the Raman spectra at 2800-3000 $\mathrm{cm}^{-1}$ (Fig. 6), with the maturity measured by Raman spectra being comparable to that by conventional biomedical methods (Fig. 1). 


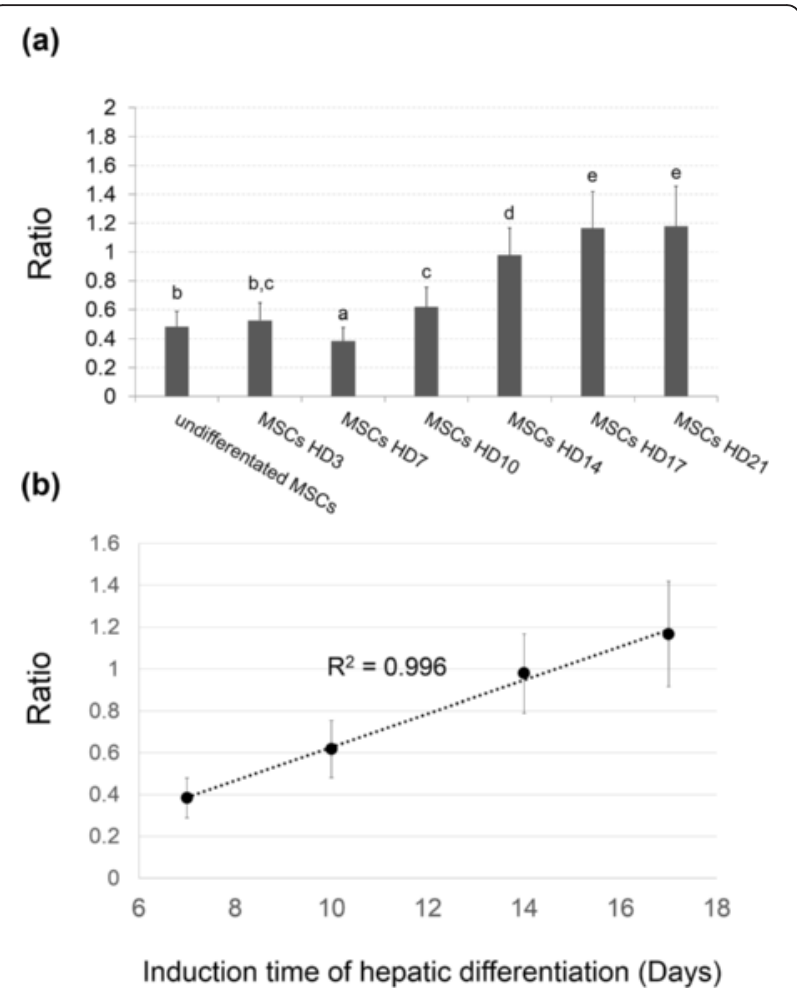

Fig. 6 a Ratio of integral area $\left(2800-3000 \mathrm{~cm}^{-1} / 400-565 \mathrm{~cm}^{-1}\right)$ and b correlation of coefficient during hepatic differentiation of the MSCs. The ratio was increased and exhibited a high degree of correlation with the induction time from day 7 to day 17 of hepatic differentiation. Groups with different alphabetical letters are significantly different, whereas groups with same alphabetical letters are not. HD Hepatic differentiation day, MSC Mesenchymal stromal cell

During hepatic differentiation, lipochrome formation was also observed in the MSC-derived hepatocytes (Fig. 2). In this study, the spectra of the MSC-derived hepatocytes (Figs. 3 and 4) exhibit lower intensity in the broad band (2800-3000 $\left.\mathrm{cm}^{-1}\right)$ with two main peaks $\left(2852 / 2898 \mathrm{~cm}^{-1}\right)$ and one peak $\left(1439 \mathrm{~cm}^{-1}\right)$ indicating less lipid accumulation compared with that of the primary hepatocytes. However, primary hepatocytes exhibited the increased intensity of the $2852 / 2898$ peaks along with the size of lipochrome (Figs. 3 and 4). It also indicates that lipids are accumulated in lipochromes of hepatocytes from adult mouse liver. One explanation is that the hepatocytes differentiated from MSCs under 21-day induction are functional, but are still relatively immature in comparison with the primary hepatocytes. Furthermore, three-dimensional scaffold structure composed of extracellular matrix support, other than induction of growth factors and cytokines, is a critical factor for hepatogenesis in liver development [37-42], but the three-dimensional scaffold is not present in this in vitro two-dimensional hepatic differentiation. Thus, the MSC-derived hepatocytes are not as mature as the primary hepatocytes in this study.

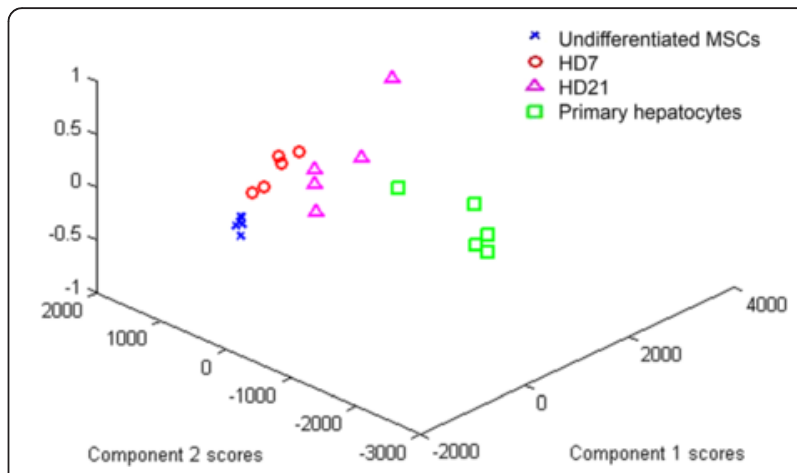

Fig. 7 PCA of hepatic differentiation of the MSCs. The PCA exhibited different scores among the undifferentiated MSCs, MSCs under hepatic differentiation at day 7 and day 21, and primary hepatocytes. HD Hepatic differentiation day, MSC Mesenchymal stromal cell

In this study, PCA of the Raman spectra for the undifferentiated MSCs and MSC-derived hepatocytes exhibited phenotypic difference on component score showing that PCA, in addition to signals at $2800-3000 \mathrm{~cm}^{-1}$, is a method to distinguish undifferentiated MSCs from MSC-derived hepatocytes (Fig. 7).

\section{Conclusion}

In summary, RS can be used as a biosensor to provide real-time information on molecular characteristics during hepatocyte differentiation of MSCs. The findings have unequivocally demonstrated the usefulness of RS in stem cell studies. The application of RS may substantially facilitate stem cell research by overcoming the limitation of current molecular biological experimental methods.

\section{Abbreviations}

DMEM: Dulbecco's modified Eagle's medium; FBS: Fetal bovine serum; IMDM: Iscove's modified Dulbecco's medium; MSC: Mesenchymal stromal cell; PAS: Periodic acid-Schiff; PBS: Phosphate-buffered saline; PCA: Principal component analysis; PCR: Polymerase chain reaction; PSG: Penicillin/ Streptomycin/ Glutamine; RS: Raman spectroscopy.

\section{Competing interests}

The authors declare that they have no competing interests.

\section{Authors' contributions}

HHW conceived and designed the experiments, performed the experiments and analyzed the data. HHW, JHH and OKL contributed reagents/materials/ analysis tools and wrote the paper. All authors read and approved the final manuscript.

\section{Acknowledgements}

This work was supported in part by the UST-UCSD International Center of Excellence in Advanced Bio-engineering (Grant Number: MOST103-2911-I009-101), under the Taiwan Ministry of Science and Technology I-RiCE Program. The authors acknowledge financial support from the Ministry of Science and Technology, Taiwan (MOST103-2314-B-010-053-MY3, MOST1032120-M-010-001, and MOST103-2321-B-010-023), the Ministry of Economic Affairs, Taiwan (103-EC-17-A-17-S1-203), the National Yang-Ming University/ Cheng Hsin General Hospital Grant (CY10405) as well as Wan Fang Hospital/ Taipei Medical University Grant (104swf03). This study was also supported by Aiming for the Top University Plan, a grant from the Ministry of Education. The authors also acknowledge the technical support provided by the Flow cytometry 
Core Facility of National Yang Ming University and the Imaging Core Facility of Nanotechnology of the UST-YMU.

\begin{abstract}
Author details
${ }^{1}$ Institute of Biophotonics, National Yang-Ming University, No. 155, Sec.2, Linong Street, Taipei 112, Taiwan. ${ }^{2}$ Center for Stem Cell Research, Wan Fang Hospital, Taipei Medical University, Taipei 116, Taiwan. ${ }^{3}$ Graduate Institute of Clinical Medicine, Taipei Medical University, Taipei, Taiwan. ${ }^{4}$ Department of Ophthalmology, Wan Fang Hospital, Taipei Medical University, Taipei, Taiwan. ${ }^{5}$ Taipei City Hospital, No. 145, Zhengzhou Road, Datong District, Taipei 10341, Taiwan. ${ }^{6}$ Institute of Clinical Medicine, National Yang-Ming University, Taipei, Taiwan. ${ }^{7}$ Stem Cell Research Center, National Yang-Ming University, Taipei, Taiwan. ${ }^{8}$ Department of Medical Research, Taipei Veterans General Hospital, Taipei, Taiwan. ${ }^{9}$ Department of Orthopaedics and Traumatology, Taipei Veterans General Hospital, Taipei, Taiwan.
\end{abstract}

\section{Received: 27 August 2015 Revised: 2 November 2015} Accepted: 4 December 2015 Published online: 11 January 2016

\section{References}

1. Raman CV. A new radiation. Indian J Phys. 1928;2:387-98.

2. Das RS, Agrawal YK. Raman spectroscopy: recent advancements, techniques and applications. Vib Spectros. 2011;57(2):163-76. doi:10.1016/j.vibspec.2011.08.003.

3. Chan JW, Lieu DK. Label-free biochemical characterization of stem cells using vibrational spectroscopy. J Biophotonics. 2009;2(11):656-68. doi:10.1002/jbio.200910041.

4. Hawi SR, Campbell WB, Kajdacsy-Balla A, Murphy R, Adar F, Nithipatikom K Characterization of normal and malignant human hepatocytes by Raman microspectroscopy. Cancer Lett. 1996;110(1-2):35-40.

5. Huang Z, McWilliams A, Lam S, English J, McLean DI, Lui H, et al. Effect of formalin fixation on the near-infrared Raman spectroscopy of normal and cancerous human bronchial tissues. Int J Oncol. 2003;23(3):649-55.

6. Galler K, Schleser F, Frohlich E, Requardt RP, Kortgen A, Bauer M, et al. Exploitation of the hepatic stellate cell Raman signature for their detection in native tissue samples. Integr Biol. 2014;6(10):946-56. doi:10.1039/c4ib00130c.

7. Guo J, Cai W, Du B, Qian M, Sun Z. Raman spectroscopic investigation on the interaction of malignant hepatocytes with doxorubicin. Biophys Chem 2009;140(1-3):57-61. doi:10.1016/j.bpc.2008.11.005.

8. Majzner K, Kochan K, Kachamakova-Trojanowska N, Maslak E, Chlopicki S, Baranska M. Raman imaging providing insights into chemical composition of lipid droplets of different size and origin: in hepatocytes and endothelium. Anal Chem. 2014;86(13):6666-74. doi:10.1021/ac501395g

9. Brauchle E, Thude S, Brucker SY, Schenke-Layland K. Cell death stages in single apoptotic and necrotic cells monitored by Raman microspectroscopy. Sci Rep. 2014;4:4698. doi:10.1038/srep04698.

10. Hung PS, Kuo YC, Chen HG, Chiang HH, Lee OK. Detection of osteogenic differentiation by differential mineralized matrix production in mesenchymal stromal cells by Raman spectroscopy. PLoS One. 2013;8(5):e65438. doi:10.1371/journal.pone.0065438.

11. Atala A. Tissue engineering, stem cells and cloning: current concepts and changing trends. Expert Opin Biol Ther. 2005;5(7):879-92. doi:10.1517/ 14712598.5.7.879.

12. Griffith $L G$, Naughton $G$. Tissue engineering-current challenges and expanding opportunities. Science (New York, NY). 2002;295(5557):1009-14. doi:10.1126/science.1069210.

13. Tuan RS, Boland G, Tuli R. Adult mesenchymal stem cells and cell-based tissue engineering. Arthritis Res Ther. 2003;5(1):32-45.

14. Wang $Y$, Chen $X$, Cao W, Shi $Y$. Plasticity of mesenchymal stem cells in immunomodulation: pathological and therapeutic implications. Nat Immunol. 2014;15(11):1009-16. doi:10.1038/ni.3002.

15. Lee KD, Kuo TK, Whang-Peng J, Chung YF, Lin CT, Chou SH, et al. In vitro hepatic differentiation of human mesenchymal stem cells. Hepatology (Baltimore, MD). 2004;40(6):1275-84. doi:10.1002/hep.20469.

16. Kuo TK, Hung SP, Chuang CH, Chen CT, Shih YR, Fang SC, et al. Stem cell therapy for liver disease: parameters governing the success of using bone marrow mesenchymal stem cells. Gastroenterology. 2008;134(7):2111-21. doi:10.1053/j.gastro.2008.03.015. 21.e1-3.
17. Sun K, Xie X, Xie J, Jiao S, Chen X, Zhao X, et al. Cell-based therapy for acute and chronic liver failures: distinct diseases, different choices. Sci Rep. 2014;4:6494. doi:10.1038/srep06494.

18. Volarevic V, Nurkovic J, Arsenijevic N, Stojkovic M. Concise review: Therapeutic potential of mesenchymal stem cells for the treatment of acute liver failure and cirrhosis. Stem Cells (Dayton, OH). 2014;32(11):2818-23. doi:10. 1002/stem.1818.

19. Peister A, Mellad JA, Larson BL, Hall BM, Gibson LF, Prockop DJ. Adult stem cells from bone marrow (MSCs) isolated from different strains of inbred mice vary in surface epitopes, rates of proliferation, and differentiation potential. Blood. 2004;103(5):1662-8. doi:10.1182/blood-2003-09-3070.

20. Klaunig JE, Goldblatt PJ, Hinton DE, Lipsky MM, Chacko J, Trump BF. Mouse liver cell culture. I. Hepatocyte isolation. In Vitro. 1981;17(10):913-25.

21. Klaunig JE, Goldblatt PJ, Hinton DE, Lipsky MM, Trump BF. Mouse liver cell culture. II. Primary culture. In Vitro. 1981;17(10):926-34.

22. Reisner LA, Cao A, Pandya AK. An integrated software system for processing, analyzing, and classifying Raman spectra. Chemometr Intell Lab Syst. 2011; 105(1):83-90. doi:10.1016/j.chemolab.2010.09.011.

23. Brunk UT, Terman A. Lipofuscin: mechanisms of age-related accumulation and influence on cell function12. Free Radic Biol Med. 2002;33(5):611-9. doi: 10.1016/S0891-5849(02)00959-0.

24. Geddes R, Stratton GC. The influence of lysosomes on glycogen metabolism. Biochem J. 1977;163(2):193-200.

25. Goldfischer S, Bernstein J. Lipofuscin (aging) pigment granules of the newborn human liver. J Cell Biol. 1969;42(1):253-61.

26. Höhn A, Jung T, Grimm S, Grune T. Lipofuscin-bound iron is a major intracellular source of oxidants: role in senescent cells. Free Radic Biol Med. 2010;48(8):1100-8. doi:10.1016/j.freeradbiomed.2010.01.030.

27. Koziolowa H, Dziezbicka E. Advances in gastroenterology. Pigment deposits in the liver. I. Lipofuscins. Pol Arch Med Wewn. 1974:52(3):305-10.

28. Tauchi $H$, Hananouchi M, Sato T. Accumulation of lipofuscin pigment in human hepatic cells from different races and in different environmental conditions. Mech Ageing Dev. 1980;12(2):183-95.

29. Ledda M, Barni L, Altieri L, Pannese E. Amount and distribution of lipofuscin in nerve and satellite cells from spinal ganglia of young adult and aged rabbits. J Submicrosc Cytol Pathol. 1999;31(2):237-46.

30. Terman A, Brunk UT. Lipofuscin. Int J Biochem Cell Biol. 2004;36(8):1400-4. doi:10.1016/j.biocel.2003.08.009.

31. Bi $Y$, He Y, Huang JY, Xu L, Tang N, He TC, et al. Induced maturation of hepatic progenitor cells in vitro. Braz J Med Biol Res. 2013;46(7):559-66. doi: 10.1590/1414-431×20132455.

32. Galat A. Study of the Raman scattering and infrared absorption spectra of branched polysaccharides. Acta Biochim Pol. 1980;27(2):135-42.

33. Kizil R, Irudayaraj J, Seetharaman K. Characterization of irradiated starches by using FT-Raman and FTIR spectroscopy. J Agric Food Chem. 2002; 50(14):3912-8.

34. Movasaghi Z, Rehman S, Rehman IU. Raman spectroscopy of biological tissues. Appl Spectros Rev. 2007:42(5):493-541. doi:10.1080/05704920701551530.

35. Aksoy C, Severcan F. Role of vibrational spectroscopy in stem cell research. Spectroscopy 2012;27(3). doi:10.1155/2012/513286.

36. Abbate $\mathrm{S}$, Conti G, Naggi A. Characterisation of the glycosidic linkage by infrared and Raman spectroscopy in the $\mathrm{C}-\mathrm{H}$ stretching region: $\mathrm{a}, \mathrm{a}-$ trehalose and a, a-trehalose-2,3,4,6,6-d10. Carbohydr Res. 1991;210:1-12. doi: 10.1016/0008-6215(91)80108-Y.

37. Kazemnejad S. Hepatic tissue engineering using scaffolds: state of the art Avicenna Journal of Medical Biotechnology. 2009;1(3):135-45.

38. Levenberg S, Huang NF, Lavik E, Rogers AB, Itskovitz-Eldor J, Langer R. Differentiation of human embryonic stem cells on three-dimensional polymer scaffolds. Proc Natl Acad Sci U S A. 2003;100(22):12741-6. doi:10. 1073/pnas.1735463100.

39. Li J, Tao R, Wu W, Cao H, Xin J, Li J, et al. 3D PLGA scaffolds improve differentiation and function of bone marrow mesenchymal stem cell-derived hepatocytes. Stem Cells Dev. 2010;19(9):1427-36. doi:10.1089/scd.2009.0415.

40. Liu T, Zhang S, Chen X, Li G, Wang Y. Hepatic differentiation of mouse embryonic stem cells in three-dimensional polymer scaffolds. Tissue Eng Part A. 2010;16(4):1115-22. doi:10.1089/ten.TEA.2009.0391.

41. Meng $X$, Leslie $P$, Zhang $Y$, Dong J. Stem cells in a three-dimensional scaffold environment. SpringerPlus. 2014;3:80. doi:10.1186/2193-1801-3-80.

42. Wu XB, Tao R. Hepatocyte differentiation of mesenchymal stem cells. Hepatobiliary Pancreat Dis Int. 2012;11(4):360-71. 